\title{
The globus pallidus receives a projection from the parafascicular nucleus in the rat
}

\author{
Anthony E. Kincaid ${ }^{1}$, John B. Penney Jr. ${ }^{2}$, Anne B. Young ${ }^{2}$ and Sarah W. Newman ${ }^{1}$ \\ ${ }^{I}$ Department of Anatomy and Cell Biology, Medical Science Building II, and ${ }^{2}$ Department of Neurology, Neuroscience Laboratory Building, \\ University of Michigan, Ann Arbor, MI 48109 (U.S.A.)
}

(Accepted 22 January 1991)

Key words: Basal ganglia; Globus pallidus; Intralaminar nucleus; Parafascicular nucleus; Fluoro-gold; Phaseolus vulgaris-leucoagglutinin

\begin{abstract}
Study of the afferents of the rat globus pallidus (GP) with Fluoro-gold, a retrograde tracer, revealed retrogradely labeled neurons in the ipsilateral parafascicular nucleus of the thalamus (PF), a previously undescribed afferent of the rat GP. We used the anterograde tracer, Phaseolus vulgaris-leucoagglutinin (PHA-L), to confirm and extend our findings. After injections of PHA-L in the PF, labeled fibers with varicosities and terminal specializations were observed in the ipsilateral GP. The topographical organization of the projection is such that lateral and ventral PF neurons project preferentially to respective parts of the GP, and medial PF neurons project primarily to the ventral GP. There were very few labeled fibers seen in the dorsal or medial GP. The presently described projection from the PF to the GP provides an additional route for the PF to influence basal ganglia circuitry.
\end{abstract}

\section{INTRODUCTION}

The mammalian basal ganglia are a group of large, subcortical nuclei that play a role in motor, motivational and cognitive behaviors ${ }^{3}$. Although it is not known how the basal ganglia function as a unit or how any one of its component nuclei influences behavior, pathologies of the different nuclei within the basal ganglia can lead to a wide spectrum of movement disorders ${ }^{2}$.

The rat globus pallidus, homologous to the lateral segment of the primate globus pallidus, is connectionally situated between the primary afferent structure of the basal ganglia, the striatum $(\mathrm{CPu})$, and the primary efferent structures of the basal ganglia, the entopeduncular nucleus (homologous to the medial segment of the primate globus pallidus) and the substantia nigra pars reticulata. Based on numerous cytoarchitectural, cytochemical and connectional studies, the rodent pallidum has been recognized to consist of the ventral pallidal (VP) and the conventional, dorsal pallidal (GP) divisions ${ }^{16}$, $17,20,49,54$. Both the VP and the GP contain high concentrations of iron in their neuropil and high concentrations of Leu-enkephalin-containing striatal afferents, but the VP is differentiated from the GP by its dense substance $P$ innervation ${ }^{16,50}$. While the majority of the VP extends rostrally, ventral to the anterior commissure, there is a small area of the caudal VP that lies dorsal to the anterior commissure, where it occupies the ventromedial part of the conventionally recognized $\mathrm{GP}^{16}$. The GP is known to have reciprocal connections with the $\mathrm{CPu}^{9,30,31,46}$, the subthalamic nucleus ${ }^{8,25,52}$ and the substantia nigra ${ }^{8,19,27}$. The rat GP has also been reported to receive a projection from the dorsal raphe ${ }^{35}$ and the pedunculopontine tegmental nucleus ${ }^{22,41}$ and to send projections to the paraventricular nucleus of the thalamus, the reticular nucleus of the thalamus and the cerebral cortex ${ }^{17,48,51}$. Through these connections the GP is in a position to play a central role in basal ganglia function.

Because the GP is a small nucleus pierced by fiber bundles and located deep within the telencephalon it has been difficult to study the afferents of this nucleus with retrograde tracers. Fluoro-gold (FG), a stilbene derivative, is reported to be optimal for studying the afferents of deep brain nuclei because it is only taken up by damaged fibers of passage, if at all, and can be iontophoretically injected into a small brain area ${ }^{37,44}$. Using FG to study the afferents of the rat GP we noted evidence for a previously undescribed projection from the parafascicular nucleus of the thalamus (PF). We confirmed this projection by injecting the anterograde tracer, Phaseolus vulgaris-leucoagglutinin (PHA-L) ${ }^{13}$, into the $\mathrm{PF}$ and noted the presence of fibers with varicosities and terminal boutons in the GP. 


\section{MATERIALS AND METHODS}

\section{Animal care and stereotaxic surgery}

Adult Sprague-Dawley rats (240-380 g; Harlan, Portage, MI) were housed in group cages and given food and water ad libitum. The animals were anesthetized with chloral hydrate $(400 \mathrm{mg} / \mathrm{kg}$, i.p.) prior to placement in a stereotaxic frame, where the head was positioned with bregma and lambda in the same horizontal plane. Injection coordinates were taken from the atlas of Paxinos and Watson ${ }^{36}$ and for the GP were: AP, -0.5 to $-1.0 \mathrm{~mm}$; ML, -3.5 to $-3.7 \mathrm{~mm}$; DV, -6.0 to $-6.5 \mathrm{~mm}$ from bregma. Variations in the coordinates were used to obtain injection sites throughout the GP. The coordinates used for the PF injections were: AP, $-3.8 \mathrm{~mm}$; ML, $-0.8 \mathrm{~mm} ; \mathrm{DV},-6.0 \mathrm{~mm}$ from bregma.

Fluoro-gold tract tracing and enkephalin immunocytochemistry

Fluoro-gold (FG; Fluoro-chromes, Englewood, CO; $2.5 \%$ in acetic acid, $\mathrm{pH} 2.9)$ was iontophoretically injected $(1-5 \mu \mathrm{A}, 7 \mathrm{~s}$ on $7 \mathrm{~s}$ off, for 4-25 min) into the GP of 19 rats using a glass micropipette with an inside tip diameter of $20-50 \mu \mathrm{m}$. Following a survival time of 4-10 days the animals were perfused with $200 \mathrm{ml}$ of $0.1 \mathrm{M}$ sodium phosphate-buffered saline (NaPBS) with $0.1 \%$ sodium nitrite, followed by $400 \mathrm{ml}$ of $4 \%$ paraformaldehyde in 0.1 $\mathrm{M}$ phosphate buffer (NaPB). The brains were postfixed in the same fixative for 1-2 $\mathrm{h}$ and transferred to $20 \%$ sucrose in NaPB overnight. Frozen coronal sections were cut at $40 \mu \mathrm{m}$ and collected in NaPB with $0.01 \%$ sodium azide. The sections were mounted onto gel-subbed slides, dehydrated in graded alcohols to xylene and coverslipped with DPX (Gallard-Schlesinger, Carle Place, NY).

To assist in the precise mapping of injection sites while using the fluorescence microscope, we took advantage of the high concentration of Leu-enkephalin (L-ENK) found in the neuropil of the $G^{16,42}$. Regularly spaced sections were processed for L-ENK fluorescence immunocytochemistry to help mark the dorsal and lateral boundaries of the GP. All incubations were preceded by 3 washes with gentle agitation in $0.02 \mathrm{M}$ potassium-phosphate buffered saline (KPBS), and incubations were carried out in the same buffer with $0.3 \%$ Triton X (Sigma, St, Louis, MO) on a rotator. The sections were incubated in primary antibody raised in rabbit (Incstar, Stillwater, MN; 1:1000 dilution) for $48 \mathrm{~h}$ at $4^{\circ} \mathrm{C}$. They were then incubated in a donkey anti-rabbit secondary antibody linked to 7-amino-4-methylooumarin-3-acetic acid (AMCA; Jackson ImmunoResearch, West Grove, PA; 1:25 dilution) for 1 h at room temperature. Finally they were mounted onto gel-subbed slides, air-dried and coverslipped with DPX.

Every other section was analyzed for FG on either a Leitz Orthoplan or Aristoplan microscope equipped with epifluorescence. FG and AMCA fluorescence was visualized using an ultraviolet filter block.

\section{PHA-L tract tracing}

PHA-L (2.5\% in $10 \mathrm{mM}$ NaPBS, pH 8.0; Vector Labs, Burlingame, CA) was iontophoretically injected $(5 \mu \mathrm{A}, 7 \mathrm{~s}$ on $7 \mathrm{~s}$ off, for $20 \mathrm{~min}$ ) into the PF of 8 rats via a glass micropipette with an inside tip diameter of 10-30 $\mu \mathrm{m}$. A control injection was placed in the adjacent mediodorsal nucleus of the thalamus in one animal. Following a 10-day survival animals were perfused with $200 \mathrm{ml}$ of 0.1 M NaPBS with $0.1 \%$ sodium nitrite followed by either $400 \mathrm{ml}$ of $4 \%$ paraformaldehyde in $0.1 \mathrm{M} \mathrm{NaPB}$ or $4 \%$ paraformaldehyde with $0.1 \%$ glutaraldehyde in $0.1 \mathrm{M} \mathrm{NaPB}$. The brains were postfixed in the same fixative for $1-2 \mathrm{~h}$ before being transferred to $20 \%$ sucrose in NaPB. Frozen coronal sections were cut at $40 \mu \mathrm{m}$ and collected in $0.02 \mathrm{M} \mathrm{KPBS}$ buffer with $0.01 \%$ sodium azide. Every third section was processed for PHA-L immunocytochemistry. These sections were washed twice before incubation in goat anti-PHAL antibody (Vector Labs; $1: 1000$ dilution) for $48 \mathrm{~h}$ at $4{ }^{\circ} \mathrm{C}$. The sections were washed 3 times and then incubated in biotinylated rabbit anti-goat antibody (Vector Labs; 1:100 dilution) for $1 \mathrm{~h}$ at room temperature. After 3 washes the sections were incubated in the Vectastain ABC solution (Vector Labs) for $1 \mathrm{~h}$ at room temperature. Fifty $\mathrm{ml}$ of freshly made $0.0125 \%$ diaminobenzidine tetrahydrochloride (DAB) solution were filtered and $\mathrm{H}_{2} \mathrm{O}_{2}$ and nickel chloride were added to final concentrations of $0.06 \%$ and $0.015 \%$, respectively. After 5-15 min in the DAB solution on a shaker table, the reaction was stopped by 5 quick rinses in distilled water, and the sections were transferred to KPBS. Finally, sections were mounted on gel-subbed slides, dehydrated in graded alcohols to xylene and coverslipped with Permount (Fisher Scientific). All washes and incubations were done in $0.02 \mathrm{M} \mathrm{KPBS}$ buffer with $2 \%$ normal rabbit serum and $0.3 \%$ Triton $X$ until the wash preceding the $A B C$ incubation; after that wash all steps were carried out in plain $0.02 \mathrm{M}$ KPBS buffer. Each wash and each incubation was carried out with gentle agitation.

Sections were viewed on a Leitz Dialux microscope under brightfield or darkfield illumination. Adjacent sections were stained with Cresyl violet and injection sites and transport were mapped with the aid of a drawing tube. Some of the immunoreacted sections were also counterstained with Cresyl violet.

\section{RESULTS}

\section{Fluoro-gold tract tracing and enkephalin immunocyto- chemistry}

Fluoro-gold injection sites appear gold and AMCA

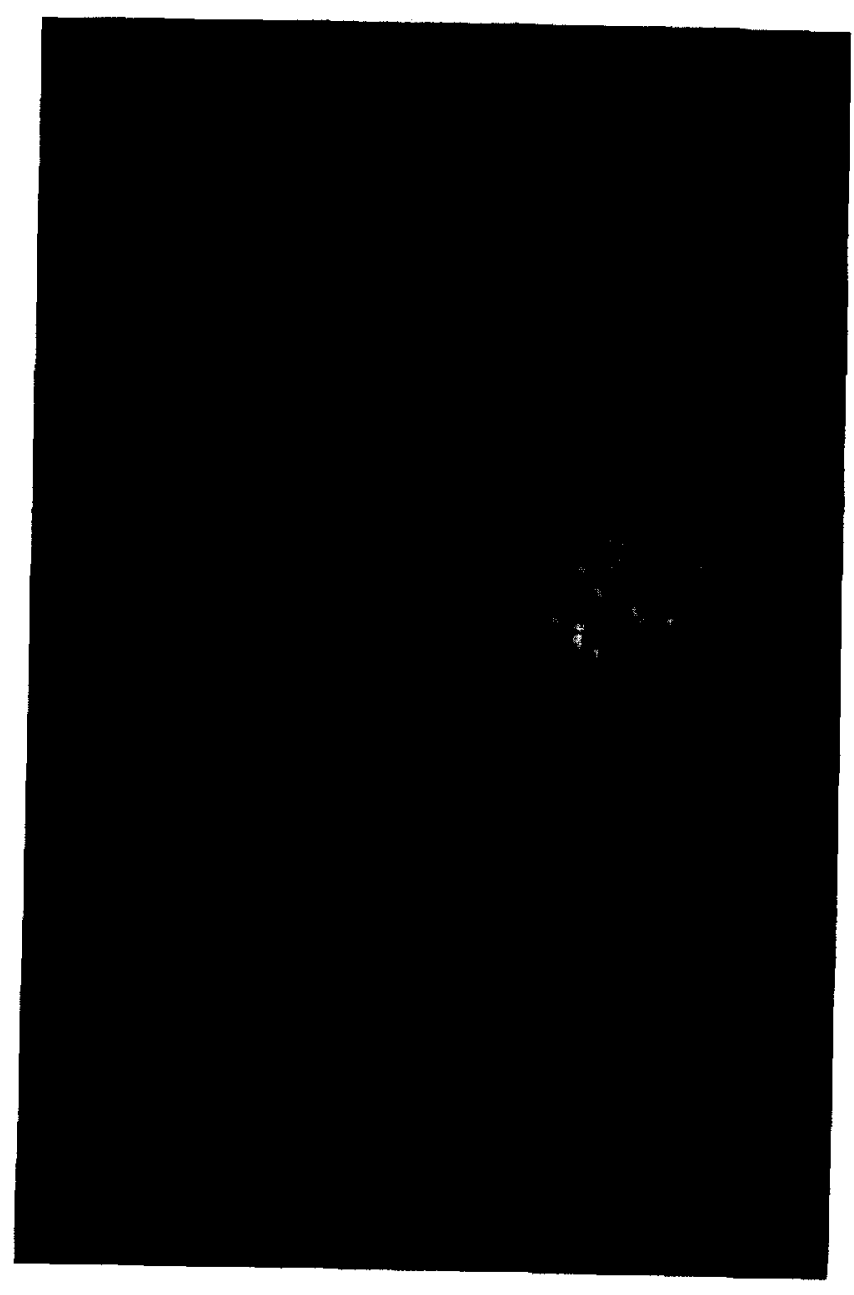

Fig. 1. Photomicrograph of a FG injection site centered in the GP, with minimal spread into the internal capsule (ic). The neuropil of the GP is immunoreactively labeled for L-ENK, which defines the striopallidal border. CPu, caudate putamen. Scale bar: $200 \mu \mathrm{m}$. 
labeled fibers appear blue under the UV filter. The injection sites were spherical, varied in size from approximately $600-1500 \mu \mathrm{m}$ in diameter (Fig. 1) and were characterized by a very small necrotic core surrounded by FG-filled neurons in diffusely labeled neuropil. In general, the use of a shorter iontophoretic injection time and lower iontophoretic current resulted in a smaller injection site, which was necessary to restrict the injection to the GP. Although these injection parameters also resulted in fewer, less intensely labeled, retrogradely filled neurons there was no difficulty in identifying labeled neurons. All retrogradely labeled neurons contained golden granules in their cytoplasm and proximal processes.

All of the injection sites resulted in retrogradely labeled neurons in the known afferents of the GP such as

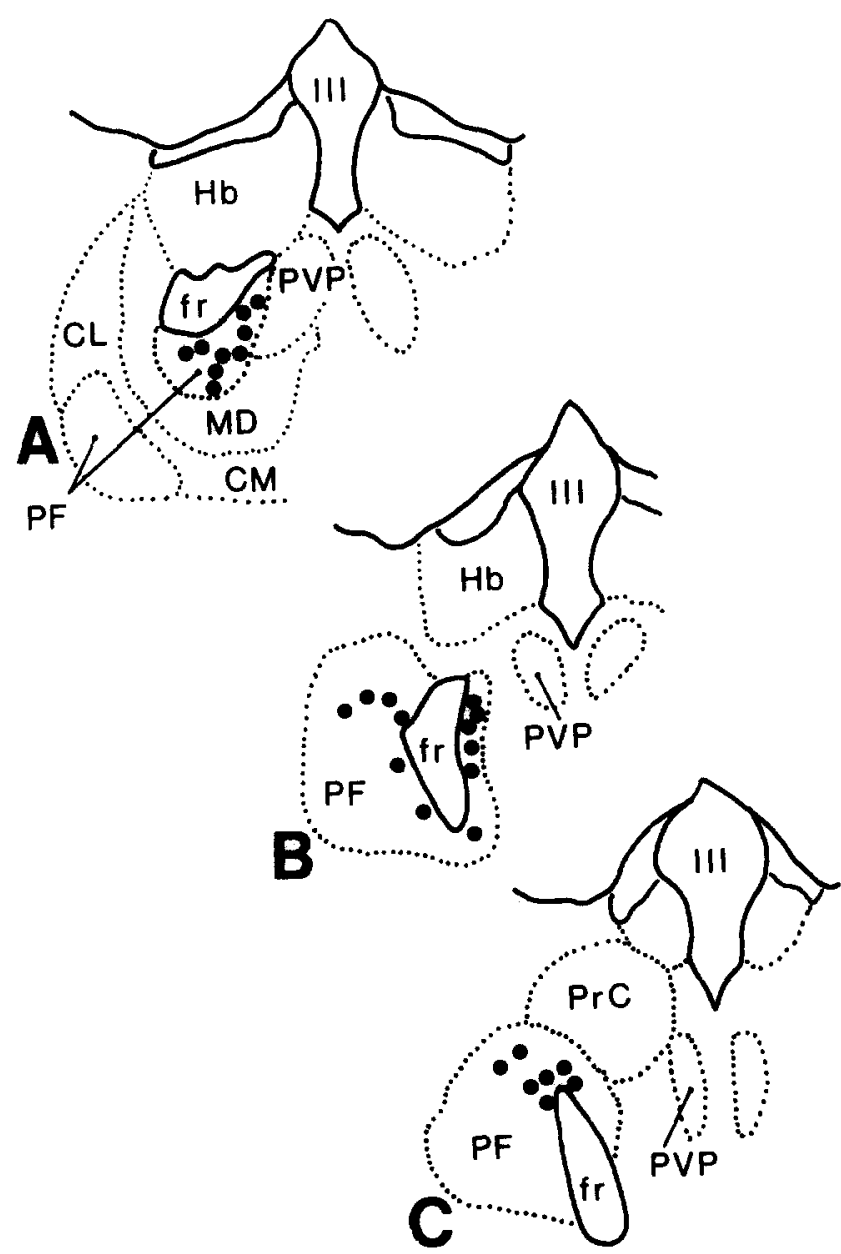

Fig. 2. Location and number of retrogradely labeled neurons in selected sections of the PF of the animal whose injection site is shown in Fig. 1. The levels of the PF in these schematic coronal sections correspond to selected levels depicted in the atlas of Paxinos and Watson; $A=-3.80 \mathrm{~mm}, \mathrm{~B}=-4.16 \mathrm{~mm}$ and $\mathrm{C}=-4.30$ $\mathrm{mm}$ from bregma. $\mathrm{CL}$, central lateral nucleus; $\mathrm{CM}$, central medial nucleus; fr, fasciculus retroflexus; $\mathrm{Hb}$, habenula; $\mathrm{MD}$, mediodorsal nucleus; PrC, precommissural nucleus; PVP, posterior paraventricular nucleus; III, third ventricle. the $\mathrm{CPu}$, the subthalamic nucleus, the substantia nigra, the pedunculopontine tegmental nucleus and the dorsal raphe. In addition to these areas, retrogradely labeled neurons were also noted in the ipsilateral PF in each case.

The AMCA-labeled L-ENK fibers effectively marked the striopallidal border allowing precise mapping of the injection site with respect to the GP (Fig. 1). The 19 animals were divided into 3 groups based on the location and extent of these injection sites. Seven brains contained injection sites that were restricted to the GP with little or no spread to surrounding nuclei. Four animals had injection sites that were centered in the GP but spread to the VP and the bed nucleus of the stria terminalis, and the remaining 8 animals had injection sites centered in the GP with some overlap into the CPu.

In the first group the injection sites were considered restricted to the GP because: (1) they did not extend outside the dorsal and lateral borders of the GP as marked with the AMCA-tagged secondary antibody for L-ENK; (2) they did not result in labeling of neurons in the somatomotor cortex, which would have indicated involvement of the $\mathrm{CPu}$; and (3) they avoided the supracommissural part of the VP. In each case these injections resulted in retrogradely labeled neurons in the PF nucleus. The labeled PF neurons were found primarily ventral and medial to the fasciculus retroflexus in 3 of the animals, primarily lateral to the fasciculus retroflexus in two of the animals and both ventromedial and lateral to the fasciculus retroflexus in the remaining two animals (Fig. 2).

Because the medial-lateral position of the GP changes with respect to midline from its rostral to caudal extent, and because the rostral and caudal portions of the nucleus are relatively narrow, it was difficult to obtain an FG injection site that was restricted to any single quadrant of the GP throughout its length. Analyzing material only from animals where the injection sites were restricted to the GP made the topographical organization of this projection difficult to interpret. While the injections restricted to the GP provided direct evidence for the projection, it was the other two injection groups that established the topography.

Injection sites centered in the GP that spread laterally into the $\mathrm{CPu}$ resulted in many retrogradely labeled neurons lateral to the fasciculus retroflexus, in the dorsolateral portion of the PF (Fig. 3A). Since the $\mathrm{CPu}$ is known to receive a dense projection from the $\mathrm{PF}^{24}$, the labeling in PF in these animals undoubtedly included neurons projecting to the $\mathrm{CPu}$ as well as the GP. In this group of injection sites, retrogradely labeled neurons were also seen in the rostral intralaminar nuclei, including the central medial and central lateral nuclei, and in the somatomotor cortex. 

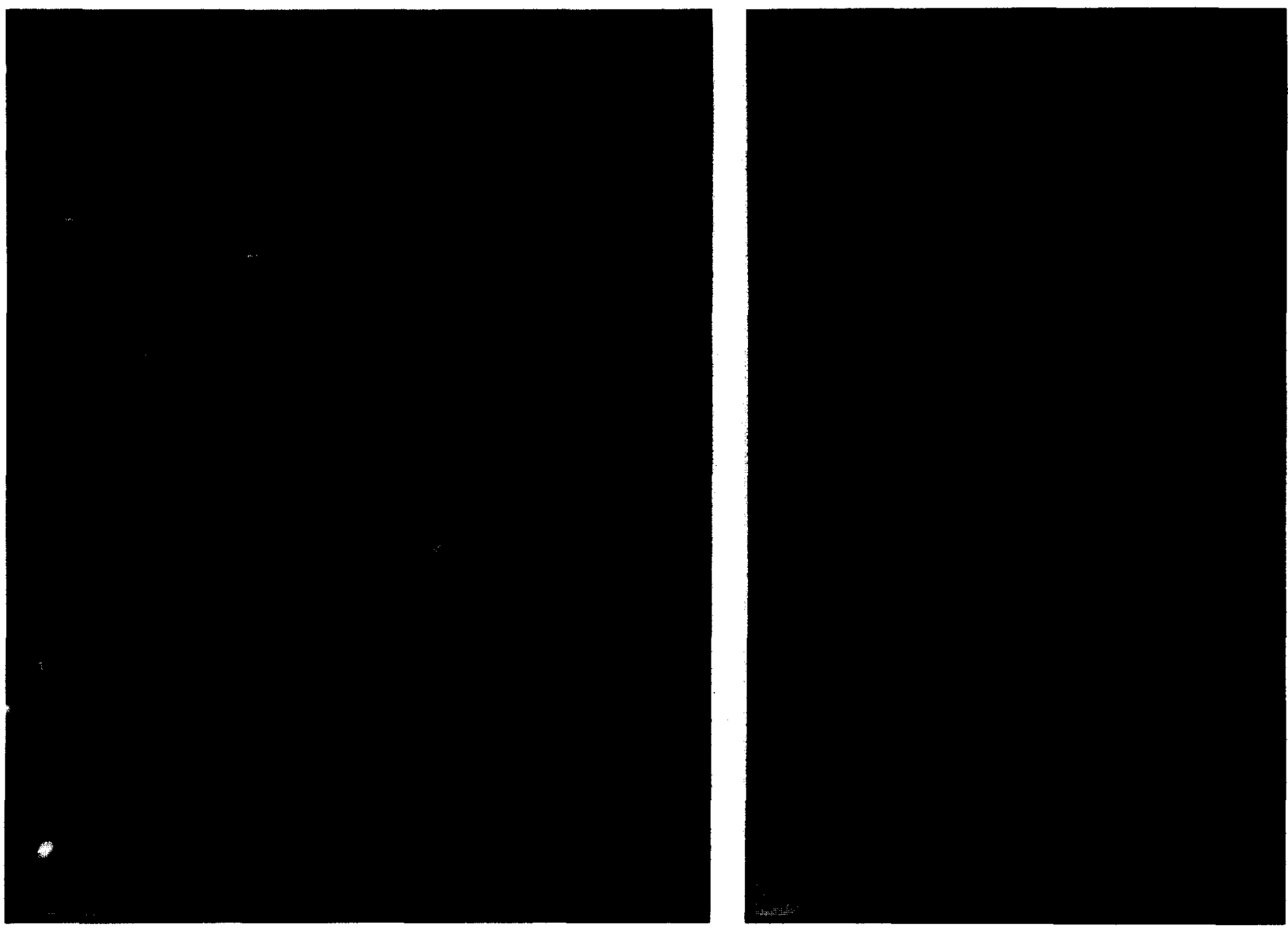

Fig. 3. Retrogradely labeled neurons in the ipsilateral PF following injections of FG centered in the lateral GP, A and medial GP, B. Midline is to the right in both photomicrographs. The lateral injection site spread into the adjacent $\mathrm{CPu}$, and the medial injection site included part of the bed nucleus of the stria terminalis and the VP. fr, fasciculus retroflexus. Scale bars: A and B, $50 \mu \mathrm{m}$.

In the third group of animals the injection sites were centered in the ventromedial GP but spread ventrally and medially to include the VP and bed nucleus of the stria terminalis. These injections resulted in retrogradely labeled neurons in the PF that were located medial to the fasciculus retroflexus (Fig. 3B). In addition to the known sources of afferents to the GP, the other areas that were noted to contain retrogradely labeled neurons in these brains included the paraventricular nucleus of the thalamus, the lateral hypothalamus, the central nucleus of the amygdala, the ventral tegmental area and the nucleus accumbens.

Several of the animals that were used in this study had injection sites that spread into the internal capsule and in each case there was no difference in the areas of the brain labeled, compared to animals where the injection site did not include the internal capsule.

\section{PHA-L tract tracing}

The PHA-L injection sites varied from 400 to $1200 \mu \mathrm{m}$ in diameter (Fig. 4A) and consisted of numerous labeled neurons and fibers, surrounded by immunoreactive neuropil. The injection sites were not completely restricted to the PF in any one animal but all could be utilized in this study because no FG-labeled neurons had been observed in the areas immediately surrounding the PF following FG injection sites restricted to the GP.

Injections of PHA-L were made into various parts of the PF nucleus in 8 animals, and in each case resulted in anterogradely labeled fibers in the ipsilateral GP. These labeled fibers were of 2 types. One type was larger in diameter with a smooth, wavy shaft and was restricted to the fiber bundles piercing the GP (Fig. 5A). These fibers did not appear to be making any synaptic contact with neurons in the GP but to be passing to more rostral structures, such as the $\mathrm{CPu}$ or cortex. They were interpreted to be fibers of passage and were seen in animals in which the injection site included the central medial or central lateral nuclei of the rostral intralaminar group, both of which are known to project to the $\mathrm{CPu}$ and cortex ${ }^{24}$. The second type of fiber was noticeably smaller in diameter and was seen to course between the 

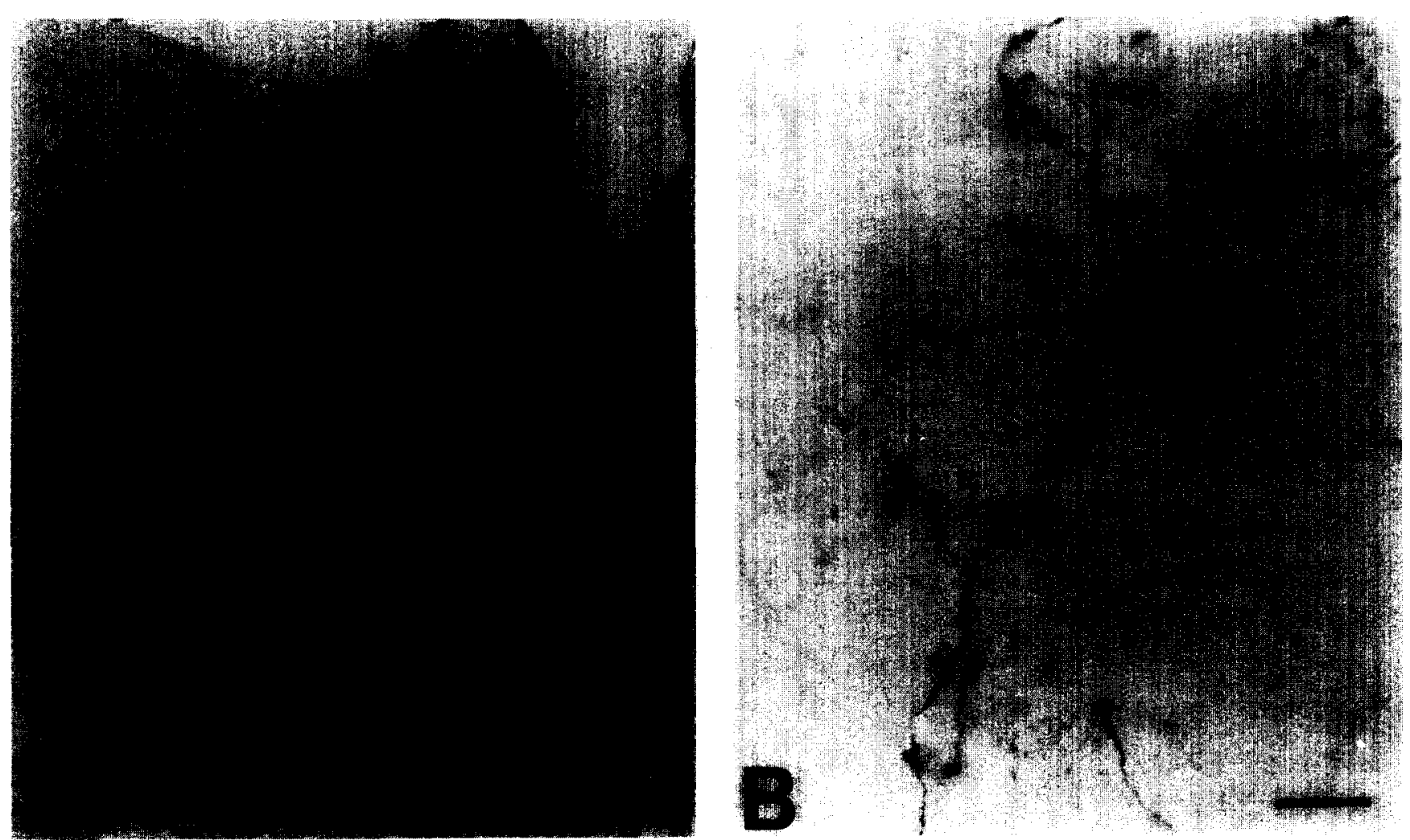

Fig. 4. A: an injection site centered lateral to the fasciculus retroflexus (fr) in the PF. B: low power view of an anterogradely labeled fiber with varicosities and apparent terminals in the lateral GP following the injection site shown in A. Scale bars: A, $250 \mu \mathrm{m} ; \mathrm{B}, 20 \mu \mathrm{m}$.

fiber bundles that pass through the GP (Fig. 5A). These fibers were noted to have numerous varicosities and terminal boutons on neurons in the GP (Figs. 4B and 5A, B). This type of fiber was observed in the GP of each brain with an injection site that included the PF nucleus.

PHA-L injection sites in the PF nucleus could be divided into 3 groups: those centered medial $(n=3)$, those centered lateral $(n=4)$ and those centered ventral $(n=1)$ to the fasciculus retroflexus.

Injections placed in the lateral half of the PF nucleus included small areas of adjacent thalamic structures such as the central lateral nucleus and the mediodorsal nucleus rostrally, and the posterior thalamic nucleus and the gustatory thalamic nucleus caudally. These injections resulted in very dense anterograde transport to the $\mathrm{CPu}$. In addition, anterogradely labeled fibers with varicosities and terminals were seen in the lateral half of the GP (Fig. 4B). Ventrolaterally centered injections resulted in anterograde transport preferentially to the ventrolateral $\mathrm{CPu}$ and GP, and dorsolaterally centered injections resulted in transport preferentially to the dorsolateral $\mathrm{CPu}$ and GP.

Injections placed in the medial half of the nucleus included adjacent thalamic structures such as the mediodorsal and paraventricular nuclei rostrally and the periventricular fiber system and precommissural nuclei cau- dally. These injections resulted in labeled fibers with varicosities and terminals primarily in the ventral part of the GP. There were very few labeled fibers seen in the dorsal or medial parts of GP in any of the animals in this study, even though one injection site was centered in the dorsomedial PF. The medial injections resulted in dense anterograde labeling in ventral areas of the striatum and in the nucleus accumbens.

When an injection site was centered ventral to the fasciculus retroflexus with minimal spread to the rostral interstitial nucleus of the medial longitudinal fasciculus, there were anterogradely labeled fibers with varicosities and terminals noted in the ventral GP (Fig. 5A,B). There was also dense anterograde label in the ventral areas of the striatum and the nucleus accumbens.

The fibers comprising this PF projection system followed two distinct routes. PHA-L labeled cells located dorsal and lateral to the fasciculus retroflexus projected their axons laterally and rostrally via the superior and intermediate thalamic radiations, as defined by Gurdijian $^{15}$, to reach the dorsal and lateral areas of the $\mathrm{GP}$ and $\mathrm{CPu}$. In contrast, labeled cells located medial and ventral to the fasciculus retroflexus sent their axons via the intermediate and inferior thalamic radiations ${ }^{15}$ to reach the ventral GP, the ventral part of the dorsal striatum and the nucleus accumbens. 

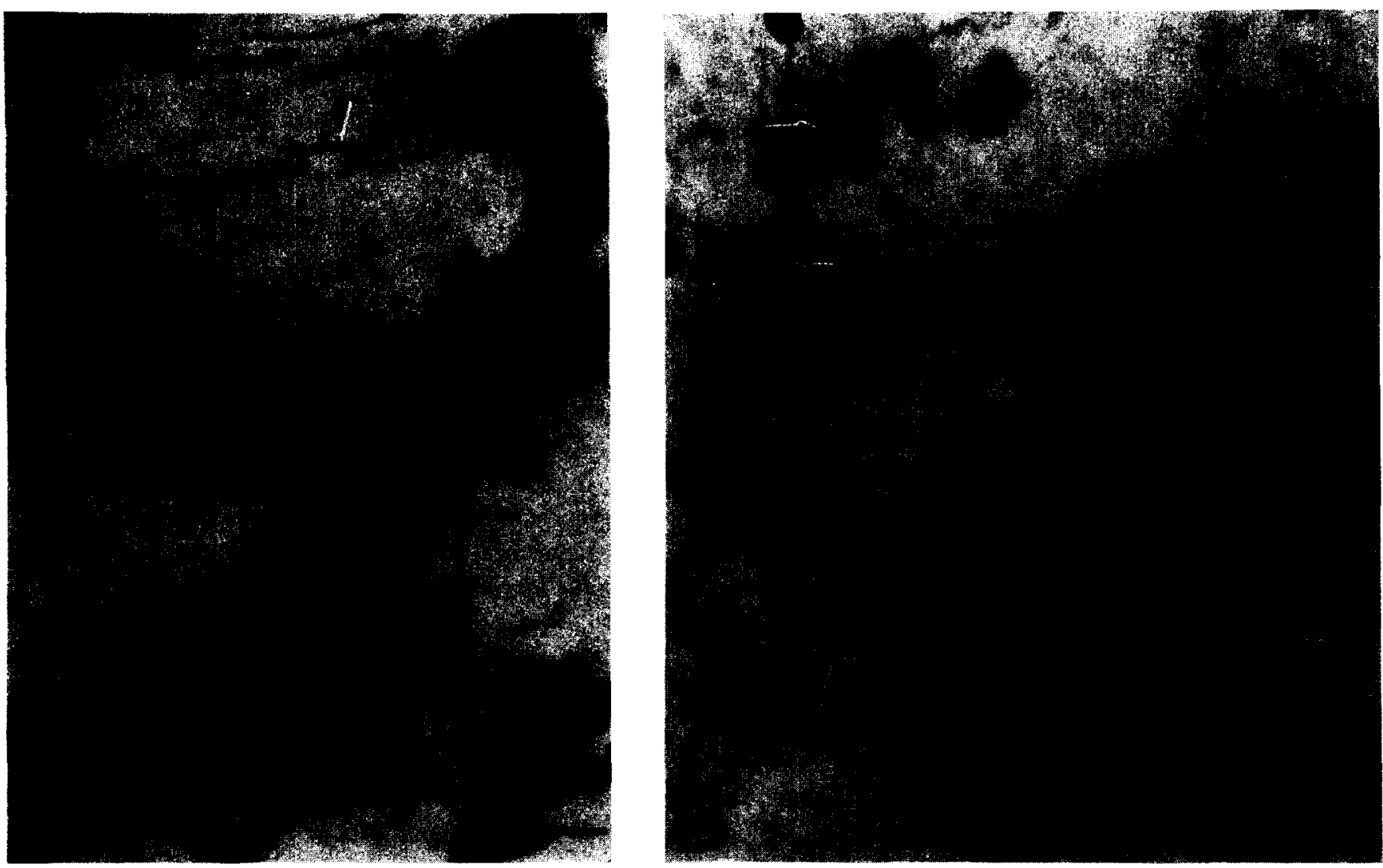

Fig. 5. High power view of anterogradely labeled fibers in the ventral GP following a PHA-L injection site centered in the ventral PF. The immunoreacted tissue is counterstained with Cresyl violet. A: labeled fibers are seen in the fiber bundles that pierce the GP and in the neuropil of the GP. Note that the labeled fibers restricted to the fiber bundles do not have varicosities or terminals (arrowhead). The labeled fibers in the neuropil, in contrast, are seen to have numerous boutons (arrows). B: labeled fibers with varicosities and apparent terminals, some of which appear to be in contact with the soma of GP neurons (arrows). Scale bars: A and B, $10 \mu \mathrm{m}$.

A control injection that was centered in the mediodorsal nucleus of the thalamus resulted in numerous anterogradely labeled fibers in the GP that were large in diameter, smooth shafted and restricted to the fiber bundles that pierce the GP. These fibers of passage did not have varicosities or terminals in the GP but could be followed through adjacent sections to the ventral agranular insular cortex where varicosities and terminal specializations were present.

\section{DISCUSSION}

The results reported here provide evidence for a topographically organized projection from the PF nucleus of the thalamus to the GP in the rat. Although definitive confirmation of this projection awaits study at the ultrastructural level, the evidence presented here strongly supports its existence.

Fluoro-gold was used in this study because it has several advantages over other fluorescent retrograde tract tracers including resistance to fading, lack of diffusion from labeled cells and excellent transport following small iontophoretic injections ${ }^{37,44}$. Further, both Pieribone and Aston-Jones ${ }^{37}$ and Schmued and Fallon $^{44}$ have investigated the uptake and transport of this tracer by fibers passing through an injection site. Both groups concluded that FG was transported only by damaged axons, following injections of large volumes or high concentrations of tracer. In this study we used a low concentration of FG and produced small injection sites, minimizing the amount of damage to fibers passing through the area.

In addition to these precautions in the use of FG, we performed experiments to directly confirm the PF projections to the GP with anterograde methods. PHA-L was chosen as an anterograde tracer because it also can be injected iontophoretically, resulting in a very small injection site, and because it provides excellent morphology of the labeled axons and terminals ${ }^{13,53}$. Following injections of PHA-L into the PF, labeled axons could be traced into the GP and varicosities and terminals were noted on GP neurons. Axonal varicosities and terminal specializations visualized at the light microscopic level and identical in appearance to those seen in this study have been demonstrated to be synaptic junctions at the electron microscopic level ${ }^{53}$. 
The fiber and terminal labeling in the GP was much less dense and less obvious than that seen in the $\mathrm{CPu}$, which may account for the fact that this projection has received little attention previously and has never been studied experimentally in the rat. A similar disparity in projection density is also reported in the dopaminergic innervation of the $\mathrm{CPu}$ and $\mathrm{GP}$ from the $\mathrm{SN}^{27}$. The $\mathrm{PF}$ and SN projections to the GP and CPu may not be as markedly unequal in density as they appear, from the point of view of individual target neurons. Rather, this discrepancy might be explained by the relative sparse packing of neurons in the neuropil of the $\mathrm{GP}^{34}$, as compared to the dense population of cell bodies of the $\mathrm{CPu}$.

The limited available evidence suggests that this projection is present in several other species. Royce and Mourey ${ }^{38}$ reported a projection from the centromedian/ parafascicular nuclear complex to the GP in cat in 1985, and Sadikot and Parent ${ }^{39}$ have recently reported a projection from the centromedian and PF nuclei to both segments of the GP in the squirrel monkey. Two other studies done in the rat have also suggested this projection. In an investigation of the afferents of the nucleus basalis magnocellularis in rat, Haring and Wang ${ }^{18}$ listed retrogradely labeled neurons in the PF nucleus following injections of horseradish peroxidase in the nucleus basalis magnocellularis or in the adjacent globus pallidus. In 1983 Staines $^{45}$ reported in his $\mathrm{PhD}$ thesis that retrogradely labeled neurons were seen in the ipsilateral PF following injections of horseradish peroxidase-wheat germ agglutinin conjugate in the GP of rats.

In this study both anterograde and retrograde tract tracing techniques indicate a topographic organization of this projection. The lateral part of the PF nucleus, lateral to the fasciculus retroflexus, projects primarily to the dorsal striatum and to the more lateral parts of the GP, which are associated with motor aspects of basal ganglia function $^{34}$. The medial PF, on the other hand, is known to project to the nucleus accumbens ${ }^{11,33}$ part of the ventral striatum, and, as demonstrated in this study, to the ventral parts of the GP. It appears that through these connections the medial PF is associated with limbic aspects of basal ganglia function ${ }^{34}$. A similar topography was noted by Beckstead ${ }^{5}$ in the cat and by Sadikot et al. ${ }^{40}$ in the primate, where the centromedian nucleus (CM)

\section{REFERENCES}

1 Albe-Fessard, D. and Besson, J.M., Convergent thalamic and cortical projections - The non-specific system. In A. Iggo (Ed), Handbook of Sensory Physiology II, Somatosensory System, Springer, Berlin, 1973, pp. 489-560.

2 Albin, R.L., Young, A.B. and Penney, J.B., The functional anatomy of basal ganglia disorders, Trends Neurosci., 12 (1989) $366-375$. projects primarily to the motor areas of the basal ganglia and the PF proper projects to the associative and limbic areas. It is generally believed that the rodent thalamus does not include a distinct $\mathrm{CM}^{23}$. Thus, it may be that the lateral $\mathrm{PF}$ in the rodent encompasses neurons with projections like those of the $\mathrm{CM}$ in species which do have a distinct CM, whereas the medial PF of the rodent may make connections more like those of the PF in species with separate PF and CM nuclei. This is consistent with Kuhlenbeck's ${ }^{26}$ proposal that the $\mathrm{CM}$, which is not present in lower mammals, represents a differentiation of the lateral PF of lower mammals.

The intralaminar nuclei, which include the $\mathrm{PF}$, have been linked to several different functional systems on anatomical and physiological grounds (for review see ref. 23). They are responsible for the cortical recruiting response, which has generated much research activity over the years but is of unknown functional significance ${ }^{29}$, 38. Perhaps related to this, Steriade ${ }^{47}$ and others have provided anatomical and physiological evidence for intralaminar thalamic involvement in the sleep-wake cycle. The caudal intralaminar nuclei, including the PF, are also associated with the pain pathway. They are known to receive spinothalamic fibers ${ }^{4,7,28}$ and to respond to painful stimuli ${ }^{1}$ and may, therefore, be important in nociception. Jones ${ }^{23}$ suggests that because of the diffuse cortical connections of the intralaminar nuclei, they may play a role in the affective, as opposed to discriminative, appreciation of pain.

The results of the present investigation and other studies emphasize the close association of the PF with the motor system. The PF receives input from several basal ganglia structures including a large input from the EP and modest inputs from the $\mathrm{SN}$ and $\mathrm{CPu}^{6,8,10,14,32}$. Considering the well known, extensive efferents of the PF to the $\mathrm{CPu}$ and the subthalamic nucleus ${ }^{5,21,49}$ and the presently described projection to the GP, the PF appears to be very closely linked to basal ganglia function ${ }^{34,40}$, and may need to be considered as part of this system as proposed by Francois et al. ${ }^{12}$.

Acknowledgements. We wish to thank Drs. Roger L. Albin and Richard A. Altschuler for critical reading of this manuscript. This work was supported by NIH Grant NS20629 to S.W.N. and NIH Grant NS19613 to A.B.Y. and J.B.P.

3 Alexander, G.E., DeLong, M.R. and Strick, P.L., Parallel organization of functionally segregated circuits linking basal ganglia and cortex, Annu. Rev. Neurosci., 9 (1986) 357-381.

4 Anderson, F.D. and Berry, C.M., Degeneration studies of long ascending fiber systems in the cat brain stem, J. Comp. Neurol., 111 (1959) 195-229.

5 Beckstead, R.M., The thalamostriatal projection in the cat, $J$. Comp. Neurol., 223 (1984) 313-346.

6 Beckstead, R.M., Domesick, V.B. and Nauta, W.J.H., Efferent 
connections of the substantia nigra and ventral tegmental area in the rat, Brain Research, 175 (1979) 191-217.

7 Bowsher, D., The termination of secondary somatosensory neurons within the thalamus of Macaca mulatta: an experimental degeneration study, J. Comp. Neurol., 117 (1961) 213-227.

8 Carter, D.A. and Fibiger, H.C., The projections of the entopeduncular nucleus and globus pallidus in rat as demonstrated by autoradiography and horseradish peroxidase histochemistry, J. Comp. Neurol., 177 (1979) 113-124.

9 Chang, H.T., Wilson, C.J. and Kitai, S.T., Single neostriatal efferent axons in the globus pallidus: a light and electron microscopic study, Science, 213 (1981) 915-918.

10 Cornwall, J. and Phillipson, O.T., Afferent projections to the parafascicular thalamic nucleus of the rat, as shown by the retrograde transport of wheat germ agglutinin, Brain Res. Rev., 20 (1988) 139-150.

11 Domesick, V.B., Further observations on the anatomy of nucleus accumbens and caudatoputamen in the rat: similarities and contrasts. In R.B. Chronister and J.F. DeFrance (Eds.), The Neurobiology of the Nucleus Accumbens, Haer Institute for Electrophysiological Research, Maine, 1981, pp. 7-39.

12 Francois, C., Percheron, G., Yelnik, J. and Tande, D., A topographic study of the course of nigral axons and of the distribution of pallidal axonal endings in the centre medianparafascicular complex of macaques, Brain Research, 473 (1988) 181-186.

13 Gerfen, C.R. and Sawchenko, P.E., An anterograde neuroanatomical tracing method that shows detailed morphology of neurons, their axons and terminals: immunohistochemical localization of an axonally transported plant lectin, phaseolusvulgaris-leucoagglutinin (PHA-L), Brain Research, 290 (1984) 219-238.

14 Gerfen, C.R., Staines, W.A., Arbuthnott, G.W. and Fibiger, H.C., Crossed connections of the substantia nigra in the rat, $J$. Comp. Neurol., 207 (1982) 283-303.

15 Gurdjian, E.S., The diencephalon of the albino rat, J. Comp. Neurol., 43 (1927) 1-114.

16 Haber, S.N. and Nauta, W.J.H., Ramifications of the globus pallidus in the rat as indicated by patterns of immunohistochemistry, Neuroscience, 9 (1983) 245-260.

17 Haber, S.N., Groenewegen, H.J., Grove, E.A. and Nauta, W.J.H., Efferent connections of the ventral pallidum: evidence of a dual striato pallidofugal pathway, J. Comp. Neurol., 235 (1985) 322-335.

18 Haring, J.H. and Wang, R.Y., The identification of some sources of afferent input to the rat nucleus basalis magnocellularis by retrograde transport of horseradish peroxidase, Brain Research, 366 (1986) 152-158.

19 Hattori, T., Fibiger, H.C. and McGeer, P.L., Demonstration of a pallido-nigral projection innervating dopaminergic neurons, $J$. Comp. Neurol., 162 (1975) 487-504.

20 Heimer, L., Switzer, R.D. and Van Hoesen, G.W., Ventral striatum and ventral pallidum. Components of the motor system?, Trends Neurosci., 5 (1982) 83-87.

21 Herkenham, M. and Pert, C.B., Mosaic distribution of opiate receptors, parafascicular projections and acetylcholinesterase in rat striatum, Nature, 291 (1981) 415-418.

22 Jackson, A. and Crossman, A.R., Nucleus tegmenti pedunculopontinus: efferent connections with special reference to the basal ganglia, studied in the rat by anterograde and retrograde transport of horseradish peroxidase, Neuroscience, 10 (1983) 725-765.

23 Jones, E.G., The intralaminar nuclei. In E.G. Jones (Ed.), The Thalamus, Plenum, 1985, pp. 606-645.

24 Jones, E.G. and Leavitt, R.Y., Retrograde axonal transport and the demonstration of non-specific projections to the cerebral cortex and striatum from thalamic intralaminar nuclei in the rat, cat and monkey, J. Comp. Neurol., 154 (1974) 349-378.

$25 \mathrm{Kita}, \mathrm{H}$. and Kitai, S.T., Efferent projections of the subthalamic nucleus in the rat: light and electron microscopic analysis with the PHA-L method, J. Comp. Neurol., 260 (1987) 435-452.

26 Kuhlenbeck, H., The human diencephalon. A summary of development, structure, function, and pathology, Confin. Neurol., Suppl., 14 (1954) 1-230.

27 Lindvall, $O$. and Bjorklund, A., Dopaminergic innervation of the globus pallidus by collaterals from the nigrostriatal pathway, Brain Research, 172 (1979) 169-173.

28 Mehler, W.R., Feferman, M.E. and Nauta, W.J.H., Ascending axon degeneration following anterolateral cordotomy. An experimental study in the monkey, Brain, 83 (1960) 718-750.

29 Morison, R.S. and Dempsey, E.W., A study of thalamocortical relations, Am. J. Physiol., 135 (1942) 281-292.

30 Nagy, J.I., Carter, D.A. and Fibiger, H.C., Anterior striatal projections to the globus pallidus, entopeduncular nucleus and substantia nigra in the rat: the GABA connection, Brain Research, 158 (1978) 15-29.

31 Nauta, H.J.W., Projections of the pallidal complex: an autoradiographic study in the cat, Neuroscience, 4 (1979) 1853-1873.

32 Nauta, W.J.H. and Mehler, W.R., Projections of the lentiform nucleus in the monkey, Brain Research, 1 (1966) 3-42.

33 Newman, R. and Winans, S.S., An experimental study of the ventral striatum of the golden hamster. I. Neuronal connections of the nucleus accumbens, J. Comp. Neurol., 191 (1980) 167-192.

34 Parent, A., Extrinsic connections of the basal ganglia, Trends Neurosci., 13 (1990) 254-258.

35 Parent, A., Descarries, L. and Beaudet, A., Organization of ascending serotonin systems in the adult rat brain. A radioautographic study after intraventricular administration of $\left({ }^{3} \mathrm{H}\right)$ 5-hydroxytryptamine, Neuroscience, 6 (1981) 115-138.

36 Paxinos, G. and Watson, C., The Rat Brain in Stereotaxic Coordinates, 2nd edn., Academic, Sydney, 1986.

37 Pieribone, V.A. and Aston-Jones, G., The iontophoretic application of fluoro-gold for the study of afferents to deep brain nuclei, Brain Research, 475 (1988) 259-271.

38 Royce, G.J. and Mourey, R.J., Efferent connections of the centromedian and parafascicular thalamic nuclei: an autoradiographic investigation in the cat, J. Comp. Neurol., 235 (1985) 277-300.

39 Sadikot, A.F. and Parent, A., Differential projections of centre median and parafascicular nuclei in squirrel monkey, Soc. Neurosci. Abstr., 15 (1989) 288.

40 Sadikot, A.F., Parent, A. and Francois, C., The centre median and parafascicular thalamic nuclei project respectively to the sensorimotor and associative-limbic striatal territories in the squirrel monkey, Brain Research, 510 (1990) 161-165.

41 Saper, C.B. and Loewy, A.D., Projections of the pedunculopontine tegmental nucleus in the rat: evidence for additional extrapyramidal circuitry, Brain Research, 252 (1982) 367-372.

42 Sar, M., Stumpf, W.E., Miller, R.J., Chang, K. and Cuatrecasas, $P$., Immunohistochemical localization of enkephalin in rat brain and spinal cord, J. Comp. Neurol., 182 (1978) 17-38.

43 Sawchenko, P.E. and Swanson, L.W., A method for tracing biochemically defined pathways in the central nervous system using combined fluorescence retrograde transport and immunohistochemical techniques, Brain Research, 210 (1981) 31-51.

44 Schmued, L.C. and Fallon, J.H., Fluoro-gold: a new fluorescent retrograde axonal tracer with numerous unique properties, Brain Research, 377 (1986) 147-154.

45 Staines, W.A., Anatomical and Histochemical Studies of the Globus Pallidus and Related Basal Ganglia Nuclei, Ph.D. Thesis, University of British Columbia, 1983.

46 Staines, W.A., Atmadja, S. and Fibiger, H.C., Demonstration of a pallidostriatal pathway by retrograde transport of HRPlabeled lectin, Brain Research, 206 (1981) 446-450.

47 Steriade, M., Mechanisms underlying cortical activation: neuronal organization and properties of the midbrain reticular core and intralaminar thalamic nuclei. In O. Pompeiano and C.A. Marsan (Eds.), Brain Mechanisms and Perceptual Awareness, Raven, New York, 1981, pp. 327-377. 
48 Sugimoto, T. and Hattori, T., Direct projections from the globus pallidus to the paraventricular nucleus of the thalamus in the rat, Brain Research, 323 (1984) 188-192.

49 Sugimoto, T., Hattori, T., Mizuno, N., Itoh, K. and Sato, M., Direct projections from the centre median-parafascicular complex to the subthalamic nucleus in the cat and rat, J. Comp. Neurol., 214 (1983) 209-216.

50 Switzer, III, R.C., Hill, J. and Heimer, L., The globus pallidus and its rostroventral extension into the olfactory tubercle of the rat: a cyto- and chemoarchitectural study, Neuroscience, 7 (1982) 1891-1904.

51 Van Der Kooy, D. and Kolb, B., Non-cholinergic globus pallidus cells that project to the cortex but not to the subthalamic nucleus in rat, Neurosci. Lett., 57 (1985) 113-118.

52 Van Der Kooy, D., Hattori, T., Shannak, K. and Hornykiewicz, O., The pallido-subthalamic projection in rat: anatomical and biochemical studies, Brain Research, 204 (1981) 253-268.

53 Wouterlood, F.G. and Groenewegen, H.J., Neuroanatomical tracing by use of Phaseolus vulgaris-leucoagglutinin (PHA-L): electron microscopy of PHA-L-filled neuronal somata, dendrites, axons and axon terminals, Brain Research, 326 (1985) 188-191.

54 Zahm, D.S., Zaborsky, L., Alheid, G.F. and Heimer, L., The ventral striatopallidothalamic projection: II. The ventral pallidothalamic link, J. Comp. Neurol., 255 (1987) 592-605. 